\title{
Colonialidad (jurídica) (Legal) coloniality
}

\author{
Pedro Garzón López \\ Universidad Autónoma Benito Juárez de Oaxaca \\ garzonpedro27@hotmail.com
}

Recibido / received: 25/02/2018

Aceptado / accepted: 10/03/2018

DOI: https://doi.org/10.20318/eunomia.2018.4164

\begin{abstract}
Resumen
La voz analiza de manera general la relación entre colonialidad y derecho, teniendo en cuenta el contexto latinoamericano y, en especial, el derecho de los pueblos indígenas. El Derecho ha sido un instrumento de la colonización desde la expansión colonial europea hasta la configuración del Estado "moderno". De ahí se deduce el estudio del derecho en clave colonial, partiendo de las aportaciones teóricas del "pensamiento decolonial latinoamericano". La estrategia consiste en situar el análisis del derecho en el contexto de la "colonialidad del saber", una perspectiva epistemológica crítica al eurocentrismo. Para este propósito nos apoyamos en el derecho indígena para cuestionar los límites del derecho moderno a la luz de la "colonialidad jurídica". Finalmente, la colonialidad jurídica es planteada como otra manera de ver el derecho sin el lente de la modernidad jurídica.
\end{abstract}

\section{Palabras clave}

Colonialismo, colonialidad jurídica, derecho, colonialidad del saber, epistemología, derecho indígena.

\begin{abstract}
The article analyzes, from a general perspective, the relationship between coloniality and law, taking into account the Latin American context and, especially, the indigenous people's law. Law has been an instrument of colonization from the European colonial expansion to the "modern" state configuration. Therefore, we approach the study of Law in a colonial sense, starting from the theoretical contributions of the "Latin American decolonial thinking". The strategy consists in placing the analysis of law in the context of the "coloniality of knowledge", a critical epistemological perspective on eurocentrism. To achieve this purpose, we use the indigenous law to question the limits of modern law in light of the "legal coloniality". Finally, the "legal coloniality" is considered as another epistemological perspective to understand the indigenous law without the "modern law" focus.
\end{abstract}

\section{Keywords}

Colonialism, legal coloniality, law, coloniality of knowledge, epistemology, indigenous law.

SUMARIO. 1. Introducción. 2. Sobre la distinción entre colonialismo y colonialidad. 3. Principales dimensiones críticas del pensamiento decolonial: colonialidad del poder, colonialidad del saber y 
colonialidad del ser. 4. La intersección entre colonialidad del saber, colonialidad jurídica y derecho indígena. 5. Consideraciones finales.

1. Introducción

El derecho ha sido históricamente un instrumento de la colonización, cuyo común denominador fue la imposición del derecho del colonizador a los pueblos o naciones sometidas. Analizar el derecho en clave colonial supone repensar el derecho a partir de una genealogía colonial, presente desde la expansión colonial europea hasta la configuración de los actuales Estados "modernos". A pesar de ello, el derecho ha sido una categoría analítica marginada tanto por los teóricos del "colonialismo interno" y de los "estudios postcoloniales" como por el "pensamiento decolonial latinoamericano", cuyas premisas críticas contra el colonialismo, el eurocentrismo y el capitalismo han girado en torno al poder, la economía, la cultura, la raza, la etnia, la clase, etc.

Evidentemente, el derecho es una categoría imprescindible en el cuestionamiento de la "colonialidad del poder", no sólo por la potente influencia del lenguaje jurídico presente en la legislación, en la Constitución y en la práctica de los operadores jurídicos del sistema jurídico dominante, sino porque proyecta un conjunto de normas, valores, principios y razonamientos que han sido normalizados por la cultura jurídica dominante.

Por tanto, en la presente voz articularemos el análisis del derecho y la colonialidad, teniendo en cuenta las aportaciones teóricas del pensamiento crítico latinoamericano ligado al denominado Proyecto Modernidad/Colonialidad/Decolonialidad. La estrategia que seguiremos para aproximarnos al estudio de la "colonialidad jurídica", consiste en utilizar el sustrato de la epistemología crítica del pensamiento decolonial, teniendo como referencia el derecho de los pueblos indígenas para cuestionar la colonialidad de la modernidad jurídica y sus prácticas hegemónicas.

De este modo, analizaremos, en primer lugar, qué se entiende por colonialidad y su distinción con respecto al colonialismo. En segundo lugar, haremos un esbozo de los principales componentes teóricos del pensamiento decolonial latinoamericano, a fin de situar nuestro objeto de estudio. En tercer lugar, abordaremos las implicaciones de la "colonialidad del saber" y la "colonialidad jurídica" en la concepción del derecho indígena, con el propósito de explicar cómo la colonialidad jurídica ha legitimado y normalizado la concepción dominante del derecho moderno occidental. Por último, haremos algunas consideraciones finales sobre los desafíos que plantea la colonialidad jurídica al (re)visar el derecho dominante desde otra perspectiva epistemológica.

2. Sobre la distinción entre colonialismo y colonialidad

El cuestionamiento del legado del colonialismo y sus secuelas opresivas en las regiones colonizadas tras el proceso de descolonización (política) ha sido objeto de estudio desde diversas perspectivas críticas. Una de las primeras tesis fue planteada en América Latina por los autores que se identificaban con el "colonialismo interno" (González Casanova, 1975, Stavenhagen, 1981; Bonfil Batalla, 1972) y aún continúa repensándose en la actualidad (González Casanova, 2006; Rivera Cusicanqui, 2010). De la misma manera, las "teorías postcoloniales" desarrolladas en el contexto anglosajón desde los años 70 han contribuido al debate, en la línea de autores como Edward Said, Homi Bhabha, Gayatri Chakravorty Spivak, etc ${ }^{1}$. A estas dos tradiciones críticas se suma, desde los años 90 , una red heterogénea de pensadores críticos

\footnotetext{
${ }^{1}$ Para una visión general, véase (Mezzadra, Spivak, Mohanty, et al., 2008); así mismo, (Said, 1990).
} 
latinoamericanos ligados al Proyecto Modernidad/Colonialidad/Decolonialidad, cuyas reflexiones críticas son próximas a las de los mencionados autores al tener un blanco común: el colonialismo, el capitalismo y el eurocentrismo. A pesar de esta proximidad, resultan relevantes las aportaciones teóricas, heurísticas y epistemológicas del pensamiento decolonial a los efectos de nuestro estudio, empezando por la distinción conceptual entre colonialismo y colonialidad, así como la perspectiva de la colonialidad del saber.

En efecto, la colonialidad es un concepto introducido en los años 90 por Aníbal Quijano (1992, 2007), y posteriormente ampliado por diferentes académicos y activistas latinoamericanos al grupo Modernidad/Colonialidad/Decolonialidad, cuya premisa básica señala que colonialidad y modernidad son dos caras de la misma moneda, solo que la modernidad se ha auto-identificado con la retórica de la emancipación a costa de encubrir la lógica de la dominación colonial (Mignolo, 2010, 2005; Grosfoguel y Castro-Gómez, 2007; Quijano, 2007; Dussel, 1994)².

Desde el pensamiento decolonial, modernidad, capitalismo y eurocentrismo se conciben como procesos coetáneos que supusieron el horizonte colonial en América Latina, y que a partir del siglo XVI se constituyeron como ejes centrales de dominación sobre la población del "nuevo mundo". Estos son los componentes básicos que están en la base de lo que Quijano denomina "colonialidad del poder", en tanto patrón de dominación/explotación/control entre colonizadores y colonizados. Este patrón de poder colonial está organizado fundamentalmente sobre de la idea de raza/etnia, y atraviesa diferentes planos y dimensiones de la vida social: poder, trabajo, conocimiento, economía, autoridad, género, etc. (Quijano, 2007).

Por tanto, un sello distintivo del grupo Modernidad/Colonialidad/Decolonialidad es la distinción entre colonialismo y colonialidad. De acuerdo con Walter Mignolo (2005, p. 33), el colonialismo hace referencia a "períodos históricos específicos y a lugares de dominio imperial", mientras que la colonialidad "denota la estructura lógica del dominio colonial". Para decirlo brevemente, el colonialismo es la ocupación territorial por medios coercitivos (militar, política, económica), mientras que la colonialidad es la imposición del imaginario cultural y epistemológico europeo hacia los sujetos colonizados. Siendo así, "el colonialismo precede a la colonialidad, la colonialidad sobrevive al colonialismo" (Maldonado-Torres, 2007, p. 131), aun cuando ambos sean términos mutuamente emparentados y nacen de un mismo parto el 12 de octubre de 1492, fecha en la que se inaugura la expansión colonial europea en las Américas.

3. Principales dimensiones críticas del pensamiento decolonial: colonialidad del poder, colonialidad del saber y colonialidad del ser

A partir de esta distinción central entre colonialismo y colonialidad se derivan al menos tres dimensiones fundamentales, donde la colonialidad sobrevive y se proyecta de manera sistemática en la experiencia del sujeto colonizado; en especial, sobre los pueblos indígenas (Garzón López, 2016). Nos referimos a la colonialidad del poder,

\footnotetext{
${ }^{2}$ En este sentido, señala Enrique Dussel, 1492 representa "la fecha del nacimiento de la modernidad; aunque su gestación - como el feto- lleve un tiempo de crecimiento intrauterino. La modernidad se originó en las ciudades europeas medievales, libres, centros de enorme creatividad. Pero 'nació' cuando Europa pudo confrontarse con 'el Otro' y controlarlo, vencerlo, violentarlo; cuando pudo definirse como un 'ego' descubridor, conquistador, colonizador de la alteridad constitutiva de la misma modernidad. De todas maneras, ese Otro no fue 'des-cubierto' como Otro, sino que fue 'en-cubierto' como 'lo Mismo' que Europa ya era desde siempre. De manera que 1492 será el momento del 'nacimiento' de la modernidad como concepto, el momento concreto del 'origen' de un 'mito' de violencia sacrificial muy particular y, al mismo tiempo, un proceso de 'en-cubrimiento' de lo no-europeo” (Dussel, 1994, pp. 7-8).
} 
la colonialidad del saber y la colonialidad del ser. Si bien estas no son las únicas coordenadas analíticas, al menos constituyen las tres "patas" principales que están en la base del pensamiento crítico latinoamericano.

La colonialidad del poder se instala desde el momento mismo en que se inaugura la expansión colonial europea en el continente de "Abya-Yala"3, y el comienzo de la jerarquización de las culturas en donde el patrón de poder de la cultura dominante atraviesa todos los ámbitos de la vida social, política, económica, epistemológica, racial, sexual, subjetividad, etc. (Quijano, 2007). Esto significa que la colonialidad del poder consiste en una relación de múltiples redes, niveles y lógicas diferentes, de modo que no existe solo una estructura jerárquica de poder, sino una heterarquía de múltiples relaciones raciales, étnicas, sexuales, epistémicas, económicas, de género, subjetividad, etc. (Grosfoguel y Castro-Gómez, 2007, p. 16). Esta colonialidad del poder es la que aún sobrevive en el caso de los pueblos indígenas, aun cuando el poder imperial haya cambiado de manos, de administradores o abandonado la posesión de colonias, manteniéndose intacta la matriz del poder colonial.

En el ámbito de la colonialidad del saber, el pensamiento decolonial se ha centrado en la crítica a las formas eurocéntricas de conocimiento. Este ha constituido la principal característica de la modernidad, según Santos, quien señala que: "EI imperialismo cultural y el epistemicidio son parte de la trayectoria histórica de la modernidad occidental" (Santos, 2002, p. 77). La colonialidad del saber se ha introducido y reproducido de manera sistemática en el conocimiento de carácter occidental a costa de marginar los saberes subalternos. De esta forma, el colonialismo europeo se consolidó no sólo a base de genocidio, sino, sobre todo, secundado por el epistemicidio. Este supuso la destrucción de los saberes indígenas mediante la imposición del imaginario cultural del colonizador como la única vía de relacionarse con la naturaleza, con el mundo social y con la propia subjetividad (Said, 2008; CastroGómez, 2005; Lander, 2003).

A partir del siglo XVIII, el conocimiento privilegiado sería el derivado del razonamiento científico; es decir, el producido en las aulas universitarias o centros de investigación bajo los presupuestos de la neutralidad, objetividad y universalidad. Cualquier otro conocimiento que no reúna tales estándares será considerado inferior, pre-científico, pre-moderno o tradicional (Grosfoguel y Santiago-Castro, 2007, p. 20). Estas son las premisas epistemológicas del pensamiento occidental que a partir del siglo XVIII contribuyeron a legitimar la ignorancia y el silenciamiento de todo conocimiento no occidental. En este sentido, el conocimiento indígena no fue la excepción.

Para disciplinar estas formas de control del conocimiento, uno de los campos privilegiados ha sido la educación, cuya función supone la interiorización del discurso científico en la producción y reproducción de conocimientos. De tal forma, el único conocimiento verdadero sería el generado por la élite científica y filosófica europea, y a partir de este parámetro debería repensarse cualquier forma de producción del conocimiento, teniendo como máximos referentes la autoridad de los pensadores

\footnotetext{
${ }^{3}$ Abya-Yala es el nombre reconocido por los pueblos indígenas para nombrar al continente americano o América Latina. Sin embargo, como bien señala Walter Mignolo, América, América latina o América sajona es una invención europea forjada en el contexto de la historia colonial a partir de 1492. Así, a comienzos del siglo XVI, "el continente no figuraba en los mapas porque no se había inventado la palabra ni había nacido la idea de un cuarto continente. El territorio existía y los pobladores también, por supuesto, pero ellos daban su propio nombre al lugar donde vivían: Tawantinsuyu a la región andina, Anáhuac a lo que en la actualidad es el valle de México y Abya-Yala a la región que hoy en día ocupa Panamá. Los pobladores originarios no conocían la extensión de lo que luego se denominó "América" (Mignolo, 2005, p. 28).
} 
occidentales (Lander, 2003; Quijano, 2007; Mignolo, 2010; Grosfoguel y CastroGómez, 2007).

Por último, la colonialidad del ser afecta a la constitución de la subjetividad y la identidad de los sujetos colonizados. Esta forma de colonialidad se proyectó en la clasificación racial y la diferenciación jerárquica de la humanidad en seres superiores e inferiores bajo diferentes categorías identitarias hetero-asignadas. Se trata, en otras palabras, de la distinción de la humanidad entre la zona del ser y la zona del no-ser, según la distinción formulada por Fanon (2009). Así, aquellos que viven en la zona del no-ser son los oprimidos, los racializados, los sub-humanos, "los condenados de la tierra"; mientras que los habitantes de la zona del ser son los privilegiados de las relaciones raciales de poder.

El efecto sistemático de la colonialidad del ser terminó socavando profundamente la identidad y la subjetividad del sujeto colonizado, convirtiéndose en un medio efectivo de control y dominación, según nos dice Charles Taylor, en la medida en que esa imagen inferior es internalizada hasta el punto de que la "propia autodespreciación se transforma en uno de los instrumentos más poderosos de su propia opresión" (Taylor, 2003, p. 44). Esto explica por qué muchas personas indígenas terminan despreciando su propia identidad para introyectar el punto de vista del grupo dominante, escondiéndose en el mestizaje o simplemente identificándose como "campesinos". El ejemplo más habitual empieza por renegar de la lengua materna, la comunidad de origen o la pertenencia étnica.

4. La intersección entre colonialidad del saber, colonialidad jurídica y derecho indígena

Habiendo expuesto de manera sucinta algunas premisas teóricas del pensamiento decolonial, consideramos que es a partir de la colonialidad del saber donde derivaremos el análisis de la "colonialidad jurídica", teniendo en cuenta el impacto de la colonialidad del saber en los conocimientos indígenas, en la ciencia jurídica y, en el derecho indígena. En este sentido, la colonialidad jurídica es concebida como una dimensión esencialmente epistemológica y, por tanto, debe ser revisada en la coyuntura entre ciencia y derecho moderno que ha subalternizado la lógica cultural que subyace en el derecho indígena.

La manera en que la colonialidad del saber se ha instalado en el imaginario de los pueblos indígenas y, por tanto, en la concepción del derecho, ha sido a través de la legitimación del conocimiento científico. Uno de los campos privilegiados ha sido la educación universitaria, cuya función comportará la interiorización del discurso científico en la producción y reproducción de conocimientos. El espacio de la universidad será concebido —siguiendo la opinión de Castro-Gómez- (2007, p. 81) "como una institución que establece las fronteras entre el conocimiento útil y el inútil, entre la doxa y la episteme, entre el conocimiento legitimo (es decir, el que goza de 'validez científica') y el conocimiento ilegitimo". En el caso que nos ocupa, la enseñanza del derecho en las facultades de Derecho constituye un espacio donde se producirá y se reproducirá la colonialidad del saber y donde los conocimientos verdaderamente jurídicos serán suministrados por la ciencia jurídica europea.

Por lo tanto, la conjugación entre ciencia y derecho ha supuesto dos instrumentos poderosos que la modernidad occidental ha utilizado para distinguir entre lo verdadero y lo falso, lo legal y lo ilegal, pues aquello que está más allá de esta dicotomía será declarado inexistente y desplazado al lado invisible de la colonialidad epistemológica. Este colonialismo epistémico de la ciencia occidental es el que será reproducido sistemáticamente en las universidades y facultades de derecho para deslegitimar cualquier realidad jurídica no occidental. Así, la negación 
científica del derecho del colonizado se fundamentará en la afirmación del derecho del colonizador como el único modelo universalmente válido para la regulación social. Esta negación radical de la co-presencia y de la simultaneidad jurídica ha sido separada por una "línea abismal" que distingue lo visible de lo invisible, según Santos (2010, pp. 29-32). A este lado de la línea visible está el monopolio de la distinción entre lo verdadero y lo falso en el campo del conocimiento científico; lo cual en el campo del derecho está determinado por lo legal y lo ilegal de acuerdo con el derecho oficial. En el otro lado de la "línea abismal" (es decir, en la zona colonial invisible), no hay conocimientos verdaderos sino creencias, magia, idolatría, comprensiones intuitivas o subjetivas que luego son objetos o materias primas de las investigaciones científicas; en este caso, en el campo del derecho estaría el vacío jurídico, considerado como lo alegal por ser un territorio sin ley.

De acuerdo con el razonamiento anterior, desde el paradigma de la ciencia del derecho occidental, el derecho indígena no puede ser calificado verdaderamente como derecho sino como un no-derecho, o, en todo caso, como "usos y costumbres", derecho consuetudinario o simplemente "fenómenos infra-jurídicos" que desde los hechos sociales pasa al terreno de lo jurídico porque recibe la intervención del Estado (Carbonnier, 1997, pp. 129-131). Esta concepción subalterna del derecho indígena ha sido normalizada por la gramática jurídica occidental en las facultades de derecho para ser incorporada en el habitus del sujeto colonial. Por ello, es común afirmar categóricamente que la "costumbre jurídica" no puede estar por encima del derecho; incluso, a menudo, este argumento es reproducido por los propios indígenas, que han pasado por un proceso de formación en dogmática jurídica. Este ejemplo pone de relieve la eficacia de la colonización epistemológica en los propios sujetos colonizados, que terminan legitimando un sistema jurídico que reproduce la hegemonía del derecho occidental en detrimento de su propio derecho. Dicho de otra manera: introducido el discurso jurídico dominante en el habitus del sujeto colonizado, el jurista indígena termina mirando su propio derecho a través del derecho occidental.

Como se advierte, la razón occidental expresada a través de la ciencia del derecho dominante ha sido un medio muy efectivo para ocultar un racismo epistemológico que niega otras experiencias jurídicas y saberes que no pasan el test de la cientificidad por ser arcaicas, primitivas, tradicionales, pre-modernas, etc. Este "racismo epistémico" (Eze, Paget y Castro-Gómez, 2008) se reproduce de manera sutil y sistemática en la formación universitaria al abstraer cualquier realidad jurídica no occidental al filtro del conocimiento eurocéntrico. Por eso, dirá Santos —con razón-: "la concepción modernista del derecho llevó a una gran pérdida de experiencia y práctica jurídica y, de hecho, legitimó un "juridicidio" masivo, esto es, la destrucción de prácticas y concepciones jurídicas que no se ajustaban al canon jurídico modernista" (2009, p. 47).

En consecuencia, la mutua interrelación entre ciencia y derecho ha legitimado no sólo una estructura jerárquica de la sociedad organizada sobre la base de un orden jurídico dominante, sino también la apropiación y expropiación de los saberes indígenas. Un ejemplo puede ilustrarse con la medicina indígena, donde la sabiduría ancestral en tratamientos curativos es usurpada por las empresas farmacéuticas para luego patentarlas bajo la protección de la propiedad intelectual. De tal forma, cuando las comunidades indígenas pretenden proteger sus conocimientos según la premisa de la propiedad intelectual, resulta que la gramática jurídica individualista no contempla la manera de garantizar la protección de tales bienes colectivos. En este caso entran en juego dos procesos constitutivos: de apropiación y de expropiación del conocimiento indígena bajo la anuencia de la ciencia y el derecho moderno. 
Ahora bien, la colonización epistemológica no sólo está implícita en la ciencia jurídica dominante, sino también en la práctica legislativa, donde predominan normas, valores y principios deducidos del discurso jurídico eurocéntrico. La colonialidad jurídica se introduce de igual manera en la hermenéutica del reconocimiento a través del cual opera la gramática jurídica occidental, al interpretar y aplicar el derecho indígena por parte de los operadores jurídicos del sistema dominante. Esto significa que la colonialidad jurídica está presente en la estructura del pensamiento y en la práctica cotidiana de los juristas del derecho estatal, pues primero se introyecta epistémicamente y luego se proyecta culturalmente en los hábitos, juicios, decisiones, razonamientos, prácticas, valores, etc.

Por último, la colonialidad jurídica es externalizada en las resoluciones jurídicas que sistemáticamente desconocen el derecho indígena al no tener en cuenta el contexto y la lógica de vida colectiva de los pueblos indígenas. En este caso, las decisiones judiciales suelen ser tomadas con "estricto apego a la legalidad", puesto que el derecho indígena es invocado correctamente en las decisiones judiciales al ajustarse al marco constitucional, convencional y legal que suscribe el reconocimiento del derecho indígena, aunque alejadas de la lógica de vida de las comunidades indígenas ${ }^{4}$. En definitiva, la colonialidad jurídica es una experiencia cotidiana que se vive y se respira en todo momento y en cualquier espacio institucional dominado por el pensamiento jurídico hegemónico, siendo expresado por diferentes formas de racismo: institucional, jurídico, epistemológico, cultural, etc.

\section{Consideraciones finales}

Consideramos que la colonialidad jurídica constituye "el punto ciego" que los operadores del sistema jurídico dominante no visibilizan, al situarse en el espacio oculto por la ciencia jurídica moderna. Ciertamente, la perspectiva "intercultural" de la justicia estatal ha contribuido para que las/los encargados de tomar decisiones judiciales tengan un ángulo de visión más allá de la tradicional ortodoxia judicial. Sin embargo, pensamos que la perspectiva intercultural no es suficiente sin un cuestionamiento fuerte del sustrato epistemológico del derecho oficial, dado que la diferencia entre el derecho indígena y el derecho estatal no solo es de naturaleza cultural, sino también colonial.

En efecto, desde la "diferencia cultural" se ha buscado armonizar el derecho indígena y el derecho estatal a través de la interpretación intercultural, a fin de evitar la imposición de la cultura jurídica dominante. Con todo, la dimensión intercultural del derecho resulta apenas la punta del iceberg, en cuyo análisis es preciso ahondar desde el enfoque de la "diferencia colonial"; de esta manera se cuestionaría la colonialidad jurídica que subyace en la gramática jurídica moderna, la cual no solo se expresa en la ciencia jurídica y en las decisiones judiciales, sino también en la legislación, en las normas, prácticas, valores, hábitos, símbolos y razonamientos de los operadores del sistema jurídico-político dominante. De ahí que el desafío de la colonialidad jurídica consista en transgredir la frontera de la modernidad jurídica para

\footnotetext{
${ }^{4}$ Aunque pueden existir cientos de casos parecidos, solo a manera de ejemplo señalamos el siguiente: El 30 de enero de 2016 fue detenido el indígena zapoteco Heliodoro Morales Mendoza por la policía federal mexicana cuando se encontraba en su comunidad de origen, en Tlacolula, Oaxaca (México). Su delito fue haber matado tres conejos en un "terreno comunal" —régimen común de propiedad colectiva en las comunidades indígenas- que había sido previamente declarado zona de reserva arqueológica por el gobierno mexicano. En esta zona, la cacería es considerada por la ley estatal como delito grave contra el medio ambiente, aun cuando se trate de animales que no están en riesgo de extinción. El indígena zapoteco lleva 6 meses en prisión, pero el juez podría condenarle a 9 años de cárcel. De acuerdo con el razonamiento jurídico invocado por los juzgadores, las decisiones tomadas al caso concreto han sido planteadas con "estricto apego a la legalidad". Fuente: periódico El Universal 02/08/2016. Disponible en: http://www.eluniversal.com.mx/articulo/estados/2016/08/2/seis-meses-en-lacarcel-por-matar-tres-conejos
} 
desvelar el racismo jurídico y epistemológico presente en el discurso jurídico moderno.

Por tanto, la colonialidad jurídica entraña una dimensión epistemológica que, a su vez, implica generar teorías y prácticas decoloniales. Esto no significa privilegiar la reflexión teórica por encima de las experiencias orales o prácticas que caracterizan el mundo indígena, ni mucho menos idealizar o aislar los saberes indígenas de todo contacto exterior, sino que se debe tener una clara vocación dialógica sin visos de universalidad. Esta apuesta dialógica supone trascender el "monotopismo autoreferente" de la modernidad hacia un diálogo entre saberes locales y entre sujetos concretos que piensan y hablan desde un lugar específico de la enunciación. Siendo así, la descolonización del derecho y la ciencia jurídica resulta una condición necesaria para afianzar una cultura de la interculturalidad, fomentar la interlegalidad entre tradiciones jurídicas diferentes y generar diálogos de saberes en un contexto de pluralismo epistemológico.

Por último, es preciso señalar que descolonizar la ciencia y el derecho moderno no significa asumir una posición esencialista del derecho indígena ni mucho menos negar la relevancia y las aportaciones de la ciencia y el derecho occidental. Más bien, de lo que se trata es vislumbrar otras zonas invisibilizadas por la ciencia moderna y ampliar el campo de visión del derecho desde otras perspectivas y lógicas culturales. En definitiva, la colonialidad jurídica es otra manera de ver el derecho sin el lente de la modernidad jurídica.

\section{Bibliografía}

BONFIL BATALLA, G. (1972), "El concepto de indio en América: una categoría de la situación colonial", Anales de Antropología Vol. IX, México, Instituto de Investigaciones Antropológicas de la UNAM, México, D. F., pp.105-124.

CARBONNIER, J. (1977), Sociología jurídica, Tecnos, Madrid.

CASTRO-GÓMEZ, S. (2005), La poscolonialidad explicada a los niños, Universidad del Cauca, Instituto Pensar-Universidad Javeriana, Popayán.

DUSSEL, E. (1994), 1492. El encubrimiento del otro: hacia el origen del mito de la modernidad, Plural editores-UMSA, La Paz.

EZE, Ch. E., PAGET, H. y CASTRO-GOMEZ, S. (2008), El color de la razón: racismo epistemológico y razón imperial, Ediciones del Signo, Buenos Aires.

FANON, F. (2009), Piel negra, máscaras blancas, Akal, Madrid.

GARZON LÓPEZ, P. (2016), Ciudadanía indígena. Del multiculturalismo a la colonialidad del poder, Prólogo de José María Sauca Cano, Centro de Estudios Políticos y Constitucionales, Madrid.

GONZÁLEZ CASANOVA, P. (1975), La democracia en México, Era, México.

GONZALEZ CASANOVA, P. (2006), "Colonialismo interno (una redefinición)". En: BORON, A., AMADEO, J. y GONZALEZ, S. (Comps.), La teoría marxista hoy, CLACSO, Buenos Aires, pp. 409-434.

GROSFOGUEL, R. y CASTRO-GÓMEZ, S. (eds.) (2007), El giro decolonial. Reflexiones para una diversidad epistémica más allá del capitalismo global, lesco-Pensar-Siglo del Hombre Editores, Bogotá.

LANDER, E. (2003) (Comp.), La colonialidad del saber: eurocentrismo y ciencias sociales, CLACSO, Buenos Aires.

MALDONADO-TORRES, N. (2007), "Sobre la colonialidad del ser: contribuciones al desarrollo de un concepto". En: GROSFOGUEL, R., y CASTRO-GÓMEZ, S. (eds.), El giro decolonial. Reflexiones para una diversidad epistémica más allá del capitalismo global, lesco-Pensar-Siglo del Hombre Editores, Bogotá, pp. 127-167.

MEZZADRA, S., SPIVAK, G., MOHANTY, Ch. et al. (2008), Estudios postcoloniales. Ensayos fundamentales, Traficantes de sueños, Madrid. 
MIGNOLO, W. (2005), La idea de América Latina. La herida colonial y la opción decolonial, Gedisa, Barcelona.

MIGNOLO, W. (2010), Desobediencia epistémica: retórica de la modernidad, lógica de la colonialidad y gramática de la descolonialidad, Ediciones del Signo, Buenos Aires.

QUIJANO, A. "Colonialidad y Modernidad/Racionalidad", Perú indígena, vol. 13, No. 29, Lima, pp. 11-20.

QUIJANO, A. (2007), "Colonialidad del poder y clasificación social". En: GROSFOGUEL R., y CASTRO-GÓMEZ, S. (eds.), El giro decolonial. Reflexiones para una diversidad epistémica más allá del capitalismo global, lesco-PensarSiglo del Hombre Editores, Bogotá, pp. 93-126.

RIVERA CUSICANQUI, S. (2010). Ch'ixinakax utxiwa: Reflexión sobre prácticas y discursos descolonizadores, Ediciones Tinta Limón, Buenos Aires.

SAID, E. W. (2008), Orientalismo, DeBolsillo, Barcelona.

SANTOS, B. de S. (2002), "Hacia una concepción multicultural de los derechos humanos", El Otro derecho, Núm. 28, pp. 59-83.

SANTOS, B. de S. (2009), Sociología jurídica crítica. Para un nuevo sentido común en el derecho, Trotta-ILSA, Madrid-Bogotá.

SANTOS, B. de S. (2010), Descolonizar el saber, reinventar el poder, Trilce-Extensión Universitaria, Montevideo.

STAVENHAGEN, R. (1981), "Siete tesis equivocadas sobre América Latina", Sociología y subdesarrollo, Nuestro tiempo, México, pp. 15-84.

TAYLOR, Ch. (2003), Multiculturalismo y la "política del reconocimiento", Fondo de Cultura Económica, Sevilla. 